\title{
UM PANORAMA ESTATÍTICO DE CUNHO ECONOMICO, SOCIAL E EDUCACIONAL DO ESTADO DO RIO GRANDE DO NORTE
}

\author{
Angélica Pollyana Queiroz de Medeiros
}

Graduada em Tecnologia em Comércio Exterior - IFRN

ange-louy@uol.com.br

Daniella Padilha Avelino Bezerra

Graduada em Tecnologia em Comércio Exterior - IFRN

danizinha_padilha@hotmail.com

Erica Lopes Araripe do Nascimento

Graduada em Tecnologia em Comércio Exterior - IFRN

ericanascimento_epsp@yahoo.com.br

João Maria Filgueira

Prof. do IFRN. Mestre em Engenharia de Produção; Especialista em Informática; Especialista em Docência do Ensino Superior; Bacharel em Estatística.

joao.filgueira@ifrn.edu.br

\section{RESUMO}

Atualmente é comum verificarmos a aplicação da Estatística nas mais diversas áreas, auxiliando na coleta, organização e interpretação de fatos numéricos, que chamamos de dados. Seu estudo é de muita relevância para a tomada de decisões coerentes com a realidade a ser analisada. $\mathrm{O}$ presente trabalho teve como meta, através de uma análise estatística, estudar as relações existentes entre as variáveis "taxa bruta de freqüência escolar", "renda per capita" e "índice de desenvolvimento humano municipal" coletadas dos cento e sessenta e seis municípios do estado do Rio Grande do Norte, no ano de 2000 - através de uma pesquisa realizada pela Organização das Nações Unidas (ONU) -, e, a partir daí, obter uma melhor visão crítica sobre o panorama econômico, educacional e social da região. Com os resultados obtidos - no geral, verificamos taxas bastante reduzidas para os municípios do estado se comparadas com a média brasileira -, pudemos verificar o grau de interferência das variáveis entre si, assim como detectar problemas gerais e específicos dentro do nosso estado, o que poderia exigir diretrizes cabíveis de ordem governamental para melhorar os resultados encontrados. E, como não poderia deixar de ser, concluímos dando sugestões sobre temas para futuros trabalhos que poderiam complementar o nosso e, assim, estender a pesquisa, de forma a agregar conhecimentos sobre a realidade do Rio Grande do Norte.

PALAVRAS-CHAVE: Estatística, Taxa Bruta de Freqüência Escolar, Renda per Capita, Índice de Desenvolvimento Humano Municipal. Rio Grande do Norte.

\section{AN OVERVIEW OF IMPRESSION RETROSTOCK ECONOMIC, SOCIAL AND EDUCATIONAL STATUS OF RIO GRANDE DO NORTE}

\begin{abstract}
Now it is common we verify the Statistics application in the most several areas, aiding in the collection, organization and interpretation of numeric facts, that called data. Your study is of a
\end{abstract}


lot of relevance for the important decisions relative to the reality to be analyzed. The present work had as goal, through a statistical analysis, to study the existent relationships among the variables "rude rate of school frequency", "per person income" and "rate of municipal human development" collected of the hundred and sixty six municipal districts of the state of Rio Grande do Norte, in the year of 2000 - through a research accomplished by the Organization of the United Nations (UN) -, and, since then, to obtain a better critical vision about the economical, educational and social situations of the area. With the obtained results - in the general, we verified quite reduced rates for the municipal districts of the state if compared with the Brazilian average -, we could verify the degree of interference of the variables to each other, as well as detecting general and specific problems inside of our state, that could demand reasonable guidelines of government order to improve the found results. And, finally, we concluded giving suggestions about themes for futures works that could complement ours and, like this, to extend the research, joining knowledge about the reality of Rio Grande do Norte.

KEY-WORDS: Statistics. Rude Rate of School Frequency. Per Person Income. Rate of Municipal Human Development. Rio Grande do Norte. 


\section{UM PANORAMA ESTATÍTICO DE CUNHO ECONOMICO, SOCIAL E EDUCACIONAL DO ESTADO DO RIO GRANDE DO NORTE}

\section{INTRODUÇÃO}

Atualmente é comum verificarmos a aplicação da Estatística nas mais diversas áreas, tais como: indústrias; hospitais e instituições de pesquisa na área de medicina; bancos e seguradoras em planejamento econômico, aplicações financeiras, seguros, comércio exterior, otimização de procedimentos de atendimento ao público; universidades e outros centros de pesquisa. Segundo Moore e Mccabe (2002), ela pode ser definida como a ciência da coleta, organização e interpretação de fatos numéricos, que chamamos de dados. Devemos aprender a ler as informações obtidas de forma crítica, levantando quais são os resultados relevantes para o estudo em questão e o que eles representam.

Com o intuito de aliar a prática e o conhecimento teórico adquirido em sala de aula no decorrer do semestre 2007.1, da disciplina de Estatística Aplicada - do curso Tecnologia em Comércio Exterior, da Instituição de ensino Centro Federal de Educação Tecnológica do Rio Grande do Norte -, a pesquisa que será desenvolvida adiante utilizará métodos empregados na disciplina para analisar de que forma as variáveis Índice de Desenvolvimento Humano Municipal (IDHM), renda per capita e taxa bruta de freqüência escolar podem relacionar-se entre si, demonstrando a influência que elas podem exercer umas nas outras. Para tal, serão utilizados dados referentes aos 166 municípios do estado do Rio Grande do Norte - estado localizado na região Nordeste do Brasil -, que foram coletados no ano de 2000 por uma pesquisa realizada pela Organização das Nações Unidas.

O trabalho foi subdividido em Contextualização e Problemática, Metodologia, Desenvolvimento Municipal, Análise dos Resultados, Considerações Finais e Referências.

\section{METODOLOGIA}

Os métodos e as técnicas utilizadas para atender à problemática do estudo seguiram as seguintes caracterizações de pesquisa: exploratório-descritiva, na forma de estudo de caso e com base em fontes bibliográficas e documentais, com auxílio, ainda, do meio digital - Internet.

A pesquisa tem caráter exploratório, pois condiz com os ensinamentos de Gil (2002, p. 41) quando este diz que este tipo de trabalho "têm como objetivo proporcionar maior familiaridade com o problema, com vistas a torná-lo mais explícito ou a constituir hipóteses". Por tal motivo, o planejamento do estudo é bastante flexível, possibilitando à consideração dos mais variados aspectos relativos ao fato estudado.

Quanto ao seu caráter descritivo, a pesquisa assim se classifica, pois é "uma caracterização do fenômeno estudado, descrevendo-se suas características, suas variáveis e as relações entre elas." (MAIA e OLIVEIRA, 2006, p. 131).

Dois estudos sobre Desenvolvimento Social foram considerados para a delimitação e justificativa do nosso Estudo, foram eles o de Leonardi(2005) e de Marcolino(2002).

A coleta de dados baseou-se em um estudo de caso sobre os índices - "taxa bruta de freqüência escolar", "renda per capita" e "índice de desenvolvimento humano municipal" - específicos do 
estado do Rio Grande do Norte. Além disso, as informações pertinentes tiveram como origem livros, pesquisas e documentos, retirados da Internet.

Foram utilizadas técnicas Estatísticas, responsáveis pela organização e apresentação dos dados (Fonseca, 1996), bem com a análise e interpretação dos dados.

Para a organização e descrição dos dados utilizamos técnicas de Estatística Descritiva (CRESPO, 2002), tais como distribuição de freqüência, moda, média, mediana, desvio-padrão, coeficiente de variação (SPIEGEL, 1993).

Para a análise e interpretação dos dados utilizamos técnicas de Análise de Correlação e Regressão, tais como diagrama de dispersão, coeficiente de correlação, grau de explicação e equação de regressão (SPIEGEL, 1993).

\section{DESENVOLVIMENTO MUNICIPAL}

O IDH, de acordo com Lucci, Branco e Mendonça (2004) é um dos instrumentos de avaliação das condições de vida da população e resulta do cruzamento de três indicadores básicos, que consideram as características fundamentais do processo de desenvolvimento humano: o PIB per capita ajustado em relação à sua capacidade de compra, expresso em dólares; o grau de conhecimento, inclusive a escolaridade; e a expectativa de vida, denominada longevidade.

A renda per capita corresponde à quantia em reais que cada habitante receberia caso o PIB fosse dividido igualmente entre toda a população (www.ipib.com.br). Ou seja: corresponde à renda média da população, por cabeça. Embora seja um índice muito útil, por se tratar de uma média esconde várias disparidades na distribuição de renda. Por exemplo, um país pode ter uma boa renda per capita, mas um alto índice de concentração de renda e grande desigualdade social. Também é possível que um país tenha uma baixa renda per capita mas não haja muita concentração de renda, não existindo, assim, grande desigualdade entre ricos e pobres.

E, de acordo com o Atlas do Desenvolvimento Humano no Brasil, de 2003, organizado pelo Programa das Nações Unidas para o Desenvolvimento (PNUD), a taxa bruta de frequência escolar é resultado de uma equação simples: o somatório de pessoas (independentemente da idade) que frequentam os cursos fundamental, secundário ou superior, dividido pela população local de faixa etária entre 7 a 22 anos de idade. Estão também incluídos na taxa os alunos de cursos supletivos de primeiro e de segundo graus, de classes de aceleração e de pós-graduação universitária. Apenas classes especiais de alfabetização são descartadas para efeito do cálculo.

Como pode ser observado a partir da leitura das definições dos índices IDH-M, renda per capita e taxa bruta de frequência escolar, eles possuem extrema importância para a realização de estudos acerca de uma dada população, pois nos fornece um panorama que envolve os aspectos econômicos, educacionais e sociais da região estudada. É bastante relevante conhecermos a situação em que se encontra a população que habita o mesmo espaço geográfico que habitamos, para que assim possamos cobrar dos nossos representantes políticos uma postura que combata os pontos falhos e que melhore ainda mais os pontos positivos.

Na mesma linha de raciocínio de pesquisa, encontram-se as teses de Leonardi (2005) e de Marcolino (2002), ambas encontradas no web site do CAPES (Coordenação de Aperfeiçoamento de Pessoal de Nível Superior). Leonardi (2005), cuja tese intitula-se Desenvolvimento Social na 
América Latina e sua relação com o crescimento econômico na década de 1990, analisa a relação existente entre o desenvolvimento social e o crescimento econômico dos países latinoamericanos, na evolução da década de 1990, buscando identificar nos paises latino-americanos que apresentaram melhores indicadores sociais reflexos sobre o crescimento do PIB per capita, além do tipo de correlação existente entre esse crescimento e cada uma das variáveis da saúde da educação, pobreza, emprego e da renda analisadas. Já Marcolino (2002), cuja tese Renda per Capita e Índice de Desenvolvimento Humano no Brasil, examina o grau de distribuição de renda e seu relacionamento com o IDH, na década de 90, no Brasil.

Outro estudo sobre o tema que merece destaque é um financiado pela Prefeitura da Cidade do Rio de Janeiro/RJ, cujo título é $O$ Índice de Desenvolvimento Humano dos Municípios das Capitais em 2000 (BREMAEKER, 2003). Esse estudo confirmou, a partir dos dados do Censo 2000/IBGE, que o IDH-M médio do conjunto dos Municípios das capitais é 54 milésimos $(0,054)$ acima da média nacional dos municípios brasileiros: os valores são 0,823 e 0,769, respectivamente.

Utilizando-se ainda a variável IDH-M como base de pesquisa, o estudo intitulado Desenvolvimento Humano do Município de Boa Vista, RR (SOUZA, L. S.; SANTOS, H. E. A. dos; e SOUZA, R. B. de L., 2008) procurou identificar qual das três variáveis - educação, longevidade e renda - melhor explica as variações do IDH-M. Utilizou-se, para este estudo, como base de dados e método de comparação os censos de 1991 e 2000, bem como as ações de políticas públicas aplicadas nesse período. Por fim, concluiu-se que a educação foi a variável que melhor explicou a evolução do IDH-M de Boa Vista, seguida da variável longevidade e da variável renda, tendendo esta última a decrescer com a chegada de imigrantes de áreas deprimidas do interior ou de outros estados.

Nesse mesmo sentido, foram as conclusões do estudo intitulado Desenvolvimento humano $e$ hierarquia urbana: uma análise do IDH-M entre as cidades mineiras (BATELLA, W. B.; DINIZ, A. M. A., 2006), quando afirmou que o subíndice educação foi o que mais colaborou para o crescimento do IDH-M entre 1991 e 2000, seguido por longevidade e renda, respectivamente. Ressalvou, ainda, que, embora a alfabetização da população tenha crescido, o que mais puxou a evolução educacional foi a taxa bruta de frequência escolar.

Por fim, sob outro ponto de vista, o trabalho de título Disparidades Regionais ou Educacionais? Um Exercício Contrafactual (DUARTE, A. J. M.; FERREIRA, P. C.; e SALVATO, M. A., 2003) traçou como objetivo investigar o impacto da escolaridade sobre a distribuição de renda do trabalho de estados/regiões do Brasil. Os autores mensuraram o quanto dos diferenciais de renda entre as Regiões Nordeste e Sudeste do Brasil, e entre os estados de Ceará e São Paulo, podem ser explicados pelas diferenças de escolaridade da população residente. Com base nos dados da Pesquisa Nacional por Amostras Domiciliares (PNAD) do ano de 1999 com as ponderações obtidas a partir dos resultados do censo 2000, a conclusão foi a seguinte: (i) mais de 50\% do diferencial de renda é explicado pelo diferencial de escolaridade; (ii) a renda do Nordeste reponderada pela escolaridade do Sudeste equivale a $93 \%$ da renda média do Brasil; e (iii) o aumento da escolaridade, mantendo-se a estrutura de salários, agrava a desigualdade de renda nas regiões/estados mais pobres. 


\section{ANÁLISE DOS RESULTADOS ESTUDO DE CASO NO RIO GRANDE DO NORTE}

Nesta seção iremos apresentar os principais resultados obtidos, seguidos de suas interpretações. Esses resultados estão estruturados da seguinte forma: inicialmente são apresentados resultados de cada variável separadamente; em seguida, são apresentados os resultados de suas correlações.

\section{TAXA BRUTA DE FREQÜÊNCIA ESCOLAR}

Os dados coletados foram organizados em uma distribuição de frequiência de treze (13) classes, conforme a Tabela 1. A partir da Tabela 1, foram calculadas várias medidas.

Tabela 1: Distribuição de Freqüiência da Taxa Bruta de Freqüência Escolar.

\begin{tabular}{|r|l|r|r|r|}
\hline \multicolumn{3}{|c|}{ Taxa bruta de frequiência escolar } & Municípios & Porcentagem \\
\hline 0,720 & ---- & 0,736 & 2 & 1,20 \\
\hline 0,736 & --- & 0,752 & 4 & 2,41 \\
\hline 0,752 & ---- & 0,767 & 5 & 3,01 \\
\hline 0,767 & ---- & 0,783 & 6 & 3,61 \\
\hline 0,783 & --- & 0,799 & 10 & 6,02 \\
\hline 0,799 & ---- & 0,815 & 37 & 22,29 \\
\hline 0,815 & --- & 0,830 & 24 & 14,46 \\
\hline 0,830 & --- & 0,846 & 36 & 21,69 \\
\hline 0,846 & ---- & 0,862 & 19 & 11,45 \\
\hline 0,862 & ---- & 0,878 & 9 & 5,42 \\
\hline 0,878 & --- & 0,893 & 5 & 3,01 \\
\hline 0,893 & --- & 0,909 & 5 & 3,01 \\
\hline 0,909 & --- & 0,925 & 4 & 2,41 \\
\hline$\sum$ & & & 166 & 100,00 \\
\hline
\end{tabular}

Fonte: Dados Próprios

O valor da média é 0,826487 , representando que a Taxa Bruta de Frequiência Escolar do Rio Grande do Norte está em torno 80\%. Ademais, os municípios mais próximos da Média são Augusto Severo, Jaçanã e Vila Flor - os três com 0,826 -, e Antônio Martins - com 0,827.

O desvio-padrão é de 0,037067, o que permite corrigir o valor da Média para mais e para menos, ou seja, a média varia entre os limites mínimo de 0,789419753 e máximo de 0,86355442. Também verificou-se que o coeficiente de variação foi de 4,484926\%. Com esses resultados, pode-se concluir que $95,56 \%$ dos municípios do RN estão próximos da média da taxa bruta de freqüência escolar, evidenciando assim uma alta representatividade da Média.

Considerando os limites mínimo e máximo da Média, entre 0,789419753 e 0,86355442, como padrão, constata-se que são 41 os municípios que estão com Taxa Bruta de Freqüência Escolar fora do padrão:

- 21 acima da média, Bodó, Florânia, Guamaré, Itajá, Itaú, Lagoa Nova, Lages, Luís Gomes, Martins, Mossoró, Natal, Patu, Pendências, Portalegre, Riacho de Santana, Santana dos Matos, São João do Sabugi, São Miguel, São Miguel de Touros, Tenente Laurentino Cruz e Viçosa; 
- e 20 abaixo da média, Baraúna, Bom Jesus, Coronel Ezequiel, Equador, Goianinha, Ielmo Marinho, Ipanguaçu, Jandaíra, Japi, Jardim de Piranhas, Lagoa de Pedras, Lagoa Salgada, Monte Alegre, Parazinho, Santa Cruz, Serra do Mel, Serra Negra do Norte, Serrinha, Tibau do Sul e Upanema.

Dentre esses municípios, a colocação de Mossoró merece destaque com 0,864; um município tão divulgado na mídia pelo seu constante crescimento (ALBUQUERQUE, 2007) foi ultrapassado nesse índice por municípios, como Luís Gomes, com 0,904, e Viçosa, que obteve o maior índice, 0,925 .

O gráfico 1, apresenta a média e suas variações para mais e para menos de acordo com o desviopadrão.

Gráfico 1: Média da Distribuição de Freqüiência da Taxa Bruta de Freqüência Escolar.

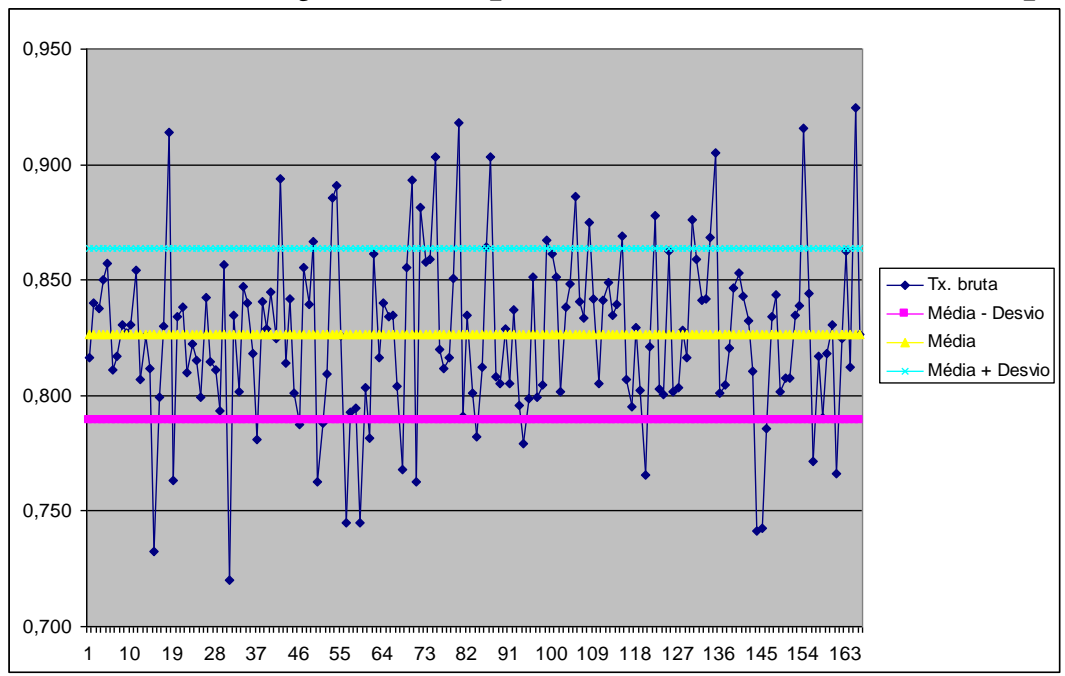

Fonte: Dados Próprios

O índice que mais se repete, o que caracteriza a Moda, está em torno de 0,809. Esse número está, nos padrões estatísticos, relativamente próximo à média, o que denota que os municípios, cujas taxas se aproximam desse valor, perfazem um total de 36, ou seja 21,69\%.

Esse resultado, comparando-se às Taxas de cidades como Natal e Outras, que estão na última classe da distribuição e significam apenas $2,41 \%$ do total, revela-se bastante baixo e denota algum problema nessa Taxa no estado, pois, há uma discrepância relevante entre aqueles resultados, baixos, e os resultados próximos ao da cidade de Natal, elevados, em torno de 0,950.

O valor da Mediana na distribuição foi de 0,827001 , o qual separa o conjunto dos dados em dois subconjuntos, um deles representa a Metade dos Municípios abaixo da Mediana e outro acima da Mediana (CRESPO, 2002). Ou seja, o cálculo da Mediana mostrou Taxas baixas para mais da metade dos municípios, já que a própria Mediana se coloca abaixo da Média e vários Municípios situados no subconjunto acima da Mediana encontram-se dentro do padrão de variação da média.

Por fim, a assimetria da distribuição foi de 0,460623. Por esse valor próximo zero (0), concluímos que a distribuição está próxima da normalidade. esse fato pode ser observado no gráfico 2, através do polígono de freqüência. 


\section{Gráfico 2: Polígono de Freqüência da Taxa Bruta de Freqüência Escolar.}

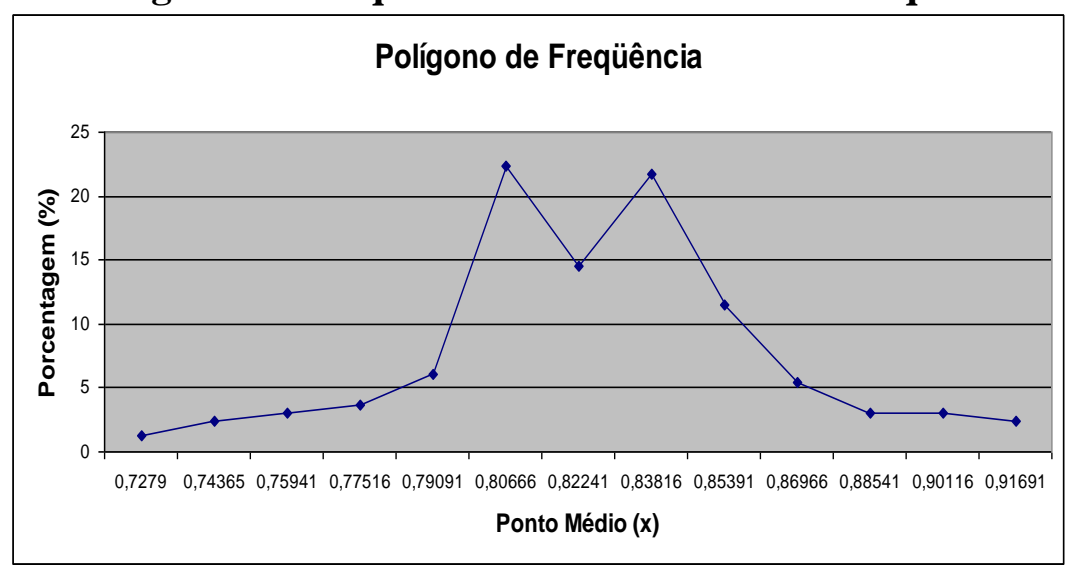

Fonte: Dados Próprios

\section{RENDA PER CAPITA}

Os cento e sessenta e seis (166) dados coletados sobre a renda per capita (em reais) foram devidamente descritos em uma distribuição de freqüência com treze (13), conforme a Tabela 2. A partir da Tabela 2, foram calculadas várias medidas.

Tabela 2: Distribuição de Freqüência da Renda Per Capita (em reais).

\begin{tabular}{|c|c|c|c|c|}
\hline \multicolumn{2}{|c|}{ Renda per capita (R\$) } & $\begin{array}{c}\text { Freqüência } \\
\text { (f) }\end{array}$ & Porcentagem \\
\hline 41,551 & ---- & 64,503 & 15 & 9,04 \\
\hline 64,503 & ---- & 87,455 & 75 & 45,18 \\
\hline 87,455 & ---- & 110,406 & 46 & 27,71 \\
\hline 110,406 & ---- & 133,358 & 12 & 7,23 \\
\hline 133,358 & ---- & 156,309 & 11 & 6,63 \\
\hline 156,309 & ---- & 179,261 & 3 & 1,81 \\
\hline 179,261 & ---- & 202,213 & 1 & 0,60 \\
\hline 202,213 & --- & 225,164 & 1 & 0,60 \\
\hline 225,164 & ---- & 248,116 & 0 & 0,00 \\
\hline 248,116 & ---- & 271,068 & 1 & 0,60 \\
\hline 271,068 & ---- & 294,019 & 0 & 0,00 \\
\hline 294,019 & ---- & 316,971 & 0 & 0,00 \\
\hline 316,971 & --- & 339,923 & 1 & 0,60 \\
\hline$\sum$ & & & 166 & 100,00 \\
\hline
\end{tabular}

Fonte: Dados Próprios

A Média da distribuição foi de 93,95293 reais; uma média muito abaixo da Média brasileira, que segundo o correio eletrônico Clipping, do Ministério do Planejamento, atingiu 9.743 reais em 2005.

O Desvio-padrão da distribuição foi de 35,74426, o que permite corrigir a Média para menos e para mais, obtendo limites mínimo e máximo para a Média. Logo, houve uma variação da média de 58,20867 reais até 129,6972 reais. Já o coeficiente de variação, que diagnostica a 
porcentagem dos dados que encontram-se próximos à média foi de aproximadamente 38,04\%, o que significa que $61,96 \%$ dos municípios estão próximos à média.

Os municípios que têm Rendas per Capita fora dos limites mínimo e máximo da Média formam um conjunto de 25 municípios:

- 18 que ultrapassaram o limite máximo, Acari, Açu, Alto do Rodrigues, Areia Branca, Caicó, Carnaúba dos Dantas, Cruzeta, Currais Novos, Extremoz, Jardim do Seridó, Macau, Mossoró, Natal, Parnamirim, Patu, Pau dos Ferros, São José do Seridó e Timbaúba dos Batistas;

- e 7 que ficaram aquém do limite mínimo, Água Nova, Coronel João Pessoa, Doutor Severiano, Japi, João Dias, Serra de São Bento e Venha-Ver.

No gráfico 3, que representa a Média da Renda per Capita, pode-se verificar essa variação fora dos limites mínimo e máximo.

Gráfico 3: Média da Distribuição de Freqüência da Renda Per Capita.

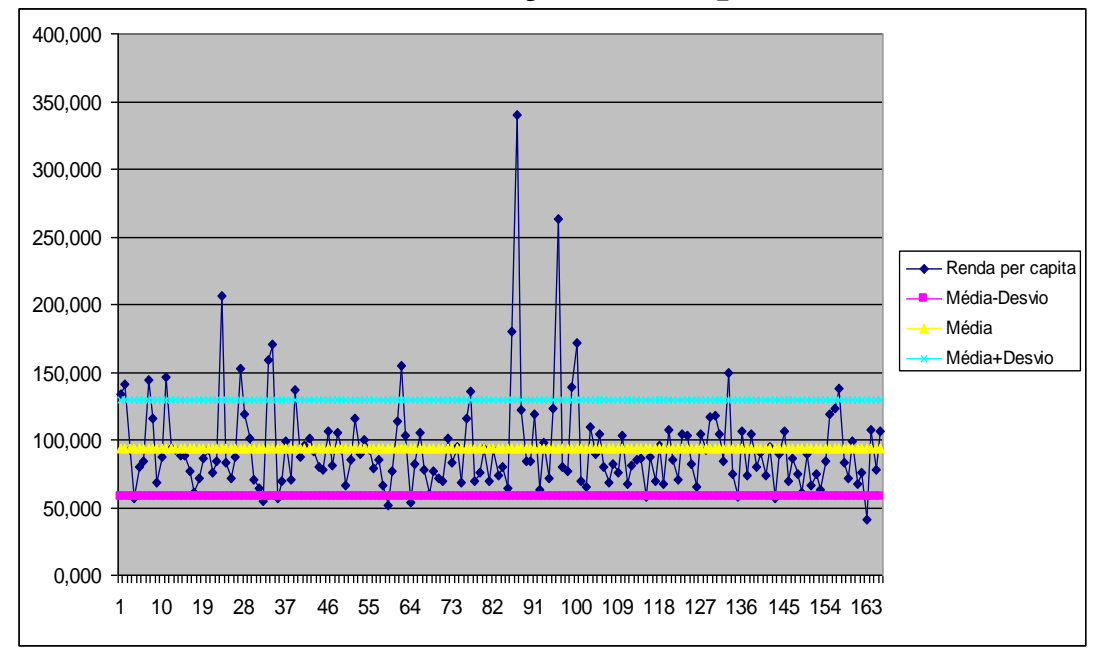

Fonte: Dados Próprios

Podemos destacar ainda no gráfico 3 a já esperada superioridade do município de Natal com maior índice de renda per capita (339,923 reais), seguido de Parnamirim (263,008 reais).

A Moda encontra-se próxima ao valor de 79,97595 reais e localiza-se logo no início da distribuição, denotando, claramente, os baixos valores de Renda per Capita de 75 municípios.

A Mediana também localiza-se no início da distribuição, e seu valor é 85,31242 reais. Como conseqüência dos valores da Moda e Mediana ocorrerem principalmente no início da distribuição, constata-se que muito Municípios com baixos valores de Renda per Capita. Ademais, detectamos uma assimetria de 6,988489, que pode ser representada pelo Polígono de Frequiência (Gráfico 4). 
Gráfico 4: Polígono de Freqüência da Renda Per Capita.

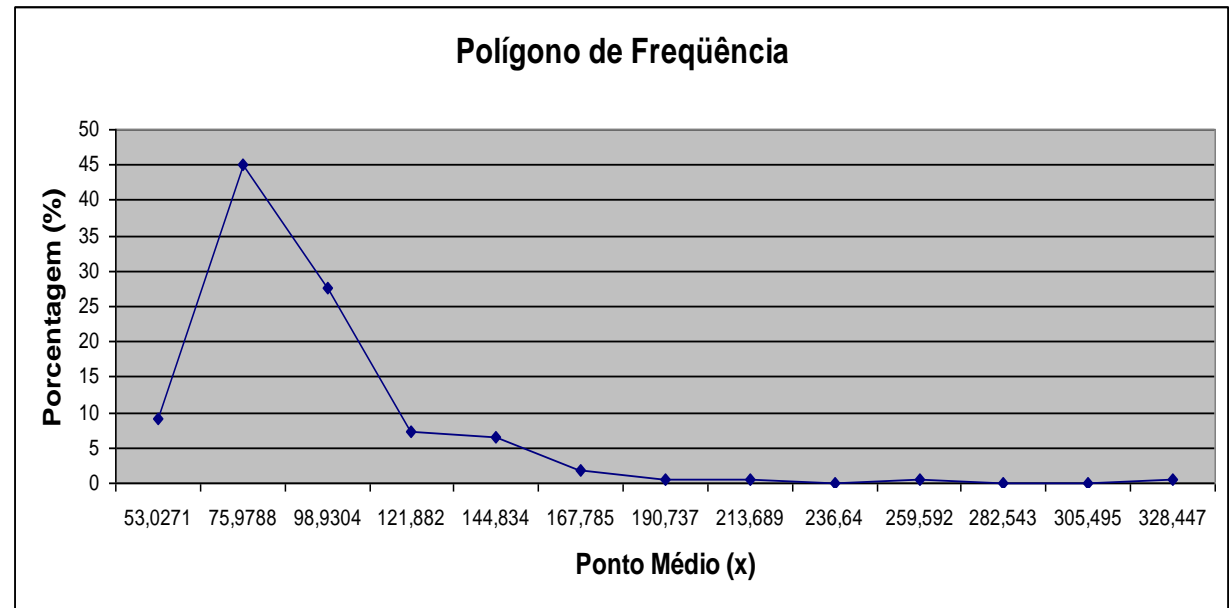

Fonte: Dados Próprios

\section{ÍNDICE DE DESENVOLVIMENTO HUMANO MUNICIPAL (IDH-M)}

A distribuição de frequiência elaborada com os dados coletados do Índice de Desenvolvimento Humano Municipal (IDH-M) foi de dividida, assim como as demais, em treze (13) classes, conforme a Tabela 3. A partir da Tabela 3, foram calculadas várias Medidas.

Tabela 3: Distribuição de Freqüiência do IDH-M.

\begin{tabular}{|c|c|c|c|c|}
\hline \multicolumn{3}{|c|}{$\begin{array}{l}\text { Índice de Des. Humano } \\
\text { Municipal (IDH-M) }\end{array}$} & \multirow{2}{*}{$\begin{array}{l}\begin{array}{c}\text { Freqüuência } \\
\text { (f) }\end{array} \\
2\end{array}$} & \multirow{2}{*}{$\begin{array}{l}\text { Porcentagem } \\
1,20\end{array}$} \\
\hline 0,544 & |-- & 0,563 & & \\
\hline 0,563 & -- & 0,582 & 9 & 5,42 \\
\hline 0,582 & -- & 0,600 & 22 & 13,25 \\
\hline 0,600 & -- & 0,619 & 26 & 15,66 \\
\hline 0,619 & -- & 0,638 & 39 & 23,49 \\
\hline 0,638 & -- & 0,656 & 28 & 16,87 \\
\hline 0,656 & -- & 0,675 & 10 & 6,02 \\
\hline 0,675 & -- & 0,694 & 11 & 6,63 \\
\hline 0,694 & -- & 0,713 & 7 & 4,22 \\
\hline 0,713 & -- & 0,731 & 6 & 3,61 \\
\hline 0,731 & -- & 0,750 & 3 & 1,81 \\
\hline 0,750 & -- & 0,769 & 2 & 1,20 \\
\hline 0,769 & -1 & 0,787 & 1 & 0,60 \\
\hline$\sum$ & & & 166 & 100,00 \\
\hline
\end{tabular}

Fonte: Dados Próprios

A média está em torno de 0,636684, e está relativamente abaixo da média brasileira, que era de 0,792 em 2005, segundo a Associação Brasileira de Editores de Livros (www.abrelivros.org.br).

De acordo com edição do Jornal O Mossoroessense, de 2003, entre as cidades Potiguares, Mossoró fica em $6^{\circ}$ lugar, tendo à sua frente as Cidades de Natal $\left(859^{\circ}\right.$ no ranking nacional $)$, 
Parnamirim $\left(1.594^{\circ}\right)$, Caicó $\left(1.695^{\circ}\right)$, Carnaúba dos Dantas $\left(2.160^{\circ}\right)$ e São José do Seridó $\left(2.225^{\circ}\right)$. Tais colocações estaduais foram confirmadas por nosso estudo.

O Desvio-padrão está em torno de 0,043519, o que significa que a Média, corrigida para mais e para menos pelo Desvio-padrão, pode variar de 0,593166 à 0,680203 . O coeficiente de variação foi de aproximadamente $6,84 \%$, um coeficiente baixo e que indica que uma porcentagem próxima a 93,16\% das Cidades encontra-se dentro desses limites da média.

Os municípios que estão acima da Média Potiguar de IDH são 25: Acari, Alto do Rodrigues, Angicos, Areia Branca, Caicó, Carnaúba dos Dantas, Cruzeta, Currais Novos, Extremoz, Grossos, Ipueira, Jardim do Seridó, Macau, Martins, Mossoró, Natal, Ouro Branco, Parelhas, Parnamirim, Pau dos Ferros, Santana do Seridó, São Gonçalo do Amarante, São João do Sabugi, São José do Seridó e Timbaúba dos Batistas.

Já os que ficaram abaixo dessa média totalizam 21: Água Nova, Bento Fernandes, Cerro Corá, Coronel João Pessoa, Espírito Santo, Ielmo Marinho, Jandaíra, Japi, João Dias, Montanhas, Parazinho, Pedra Grande, Pedra Preta, Pureza, Ruy Barbosa, São Miguel de Touros, Senador Elói de Souza, Taipu, Triunfo Potiguar, Upanema e Venha-Ver.

Dessa relação de Cidades merece destaque a posição de Venha-Ver. Pois essa ficou em último lugar na Classificação estadual:

- tanto na classificação de Renda per Capita, com 41,551 reais, o que representa 298,372 reais a menos que Natal, que possui a maior renda;

- quanto na classificação de IDH-M, com valor 0,544, que representa uma diferença de 0,243 para o primeiro lugar, Natal.

A Média do IDH-M e suas variações para mais e para menos de acordo com o desvio-padrão encontram-se no Gráfico 5.

\section{Gráfico 5: Média da Distribuição de Freqüência do IDH-M.}

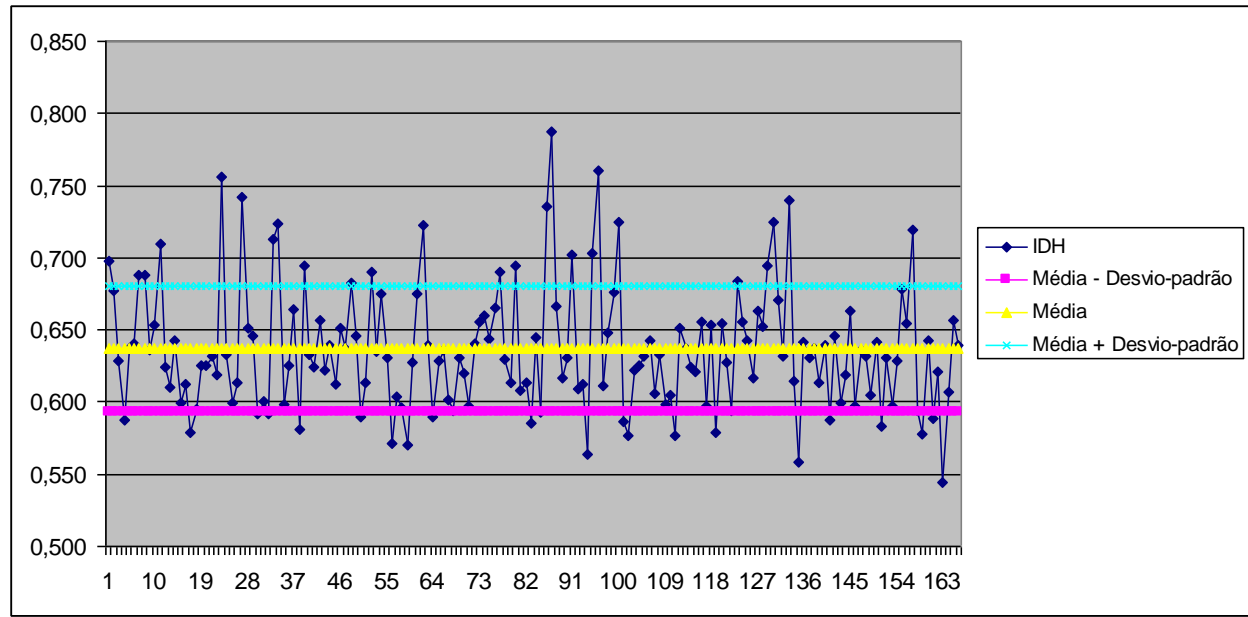

Fonte: Dados Próprios 
A classe Modal, que é aquela com a maior frequiência, destacou-se como sendo a quinta classe, e o valor da Moda foi de 0,629125. A Mediana também está nessa classe e seu valor é de 0,630504 .

Como é perceptível, os valores da Média, Moda e Mediana foram muito próximos, 0,636684, 0,629125 e 0,630504, respectivamente, o que denota uma distribuição com comportamento próximo daquele comportamento Simétrico ou Normal, característica desejável que tem como pré-requisito a igualdade entre essas três medidas de posição.

Desse fato, destacamos a baixa Assimetria de 0,173708, que está representada pelo Gráfico 6.

Gráfico 6: Polígono de Freqüência do IDH-M.

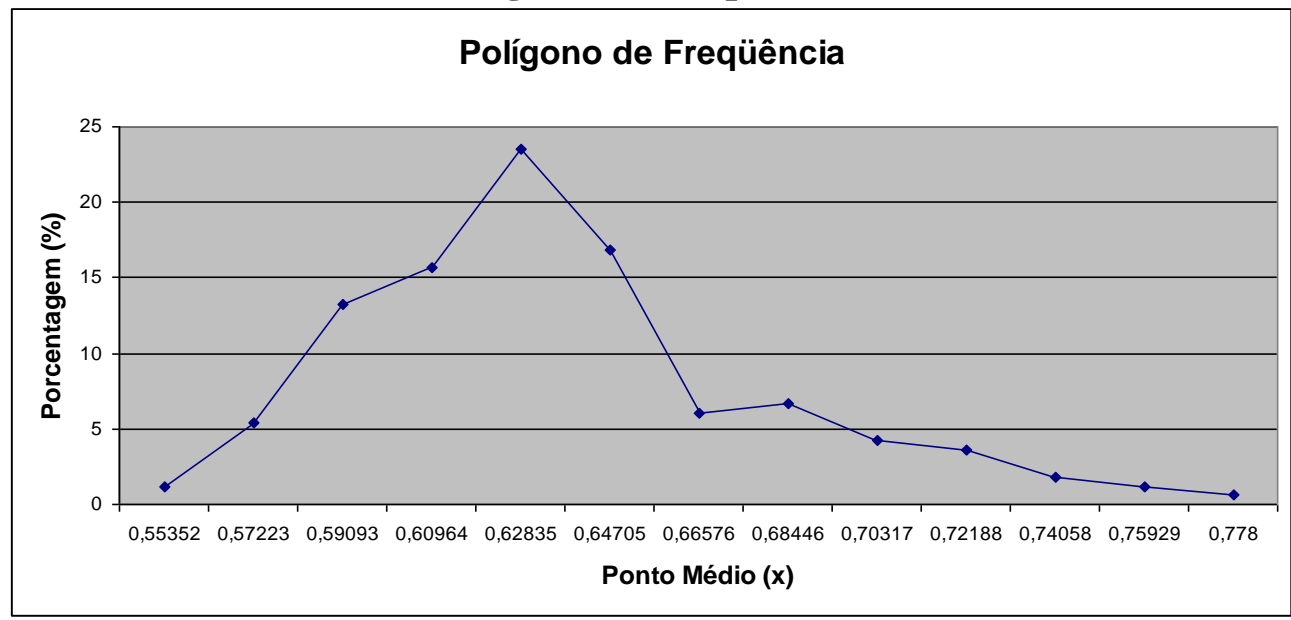

Fonte: Dados Próprios

O Gráfico 6 mostra que poucos municípios do estado possuem IDH superiores à média 0,636684, o que é preocupante para o desenvolvimento social do Rio Grande do Norte.

\section{CORRELAÇÕES}

Finalmente, é importante também analisarmos as correlações existentes entre as variáveis, de forma a atender ao questionamento proposto por nossa problemática. As análises estão estruturadas em três grupos:

- Taxa Bruta de Frequiência Escolar (X) e o IDH-M (Y);

- Taxa Bruta de Freqüência Escolar (X) e a Renda per Capita (Y); e

- IDH-M (X) e a Renda per Capita (Y).

No estudo da Correlação existente entre as varáveis da Taxa Bruta de Freqüência Escolar e o IDH-M do estado do Rio Grande do Norte, constatamos que para essas duas variáveis a covariância $(X, Y)$ foi de 9,453701; o desvio de X, 6,126755; e o desvio de Y, 7,110527, o que resultou numa Correlação de 0,217005, um resultado baixíssimo, próximo a zero. 
Com esse resultado, concluímos que a Taxa Bruta de Freqüência Escolar pouco interfere no IDH-M. Podemos constatar isso no Gráfico 7, com o diagrama de dispersão formulado, que denotou uma correlação muito baixa.

Gráfico 7: Diagrama de Dispersão da Correlação entre Taxa Bruta de Frequiência Escolar e IDH-M.

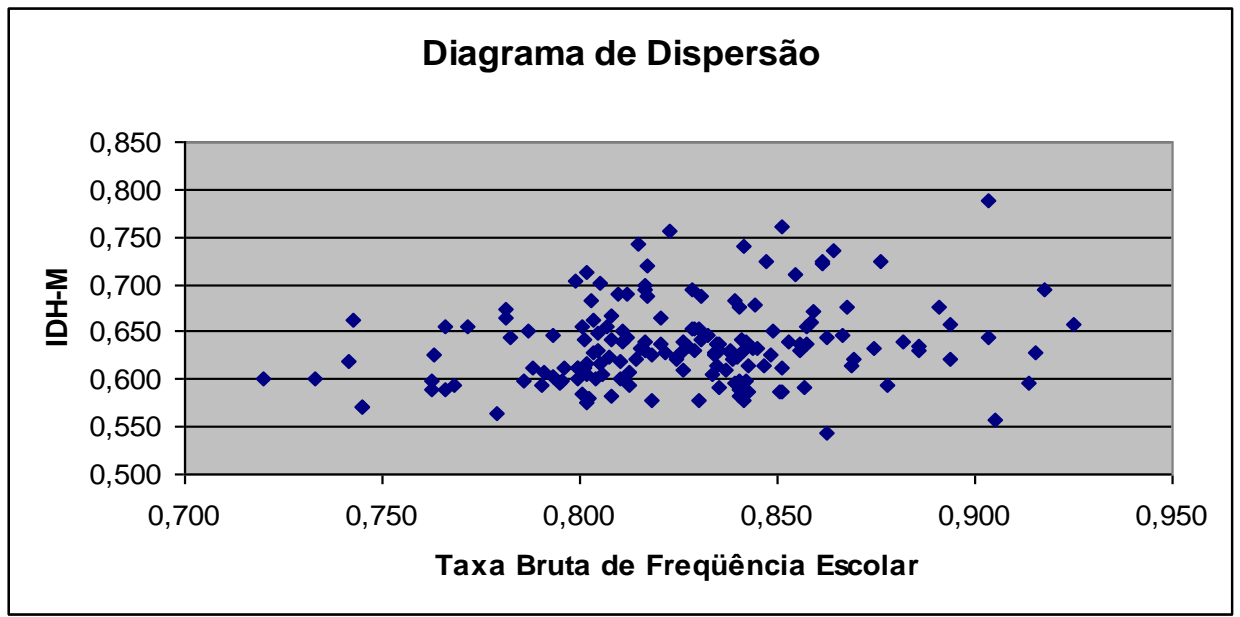

Fonte: Dados Próprios

Ao calcular a Correlação entre as variáveis Taxa Bruta de Freqüência Escolar e a Renda per Capita, detectamos uma covariância $(\mathrm{X}, \mathrm{Y})$ de 5233,197, um desvio de X de 6,126755 e um desvio de Y de 5891,057, o que resultou numa correlação novamente baixíssima de 0,144992.

Diante desses resultados podemos afirmar que a Taxa Bruta de Freqüência Escolar do RN não interfere tanto na Renda per Capita da população do Estado.

O Gráfico 8, com o diagrama de dispersão mostra um aspecto não-linear, comprovando o seu baixo valor.

\section{Gráfico 8: Diagrama de Dispersão da Correlação entre Taxa Bruta de Freqüência Escolar Renda Per Capita}

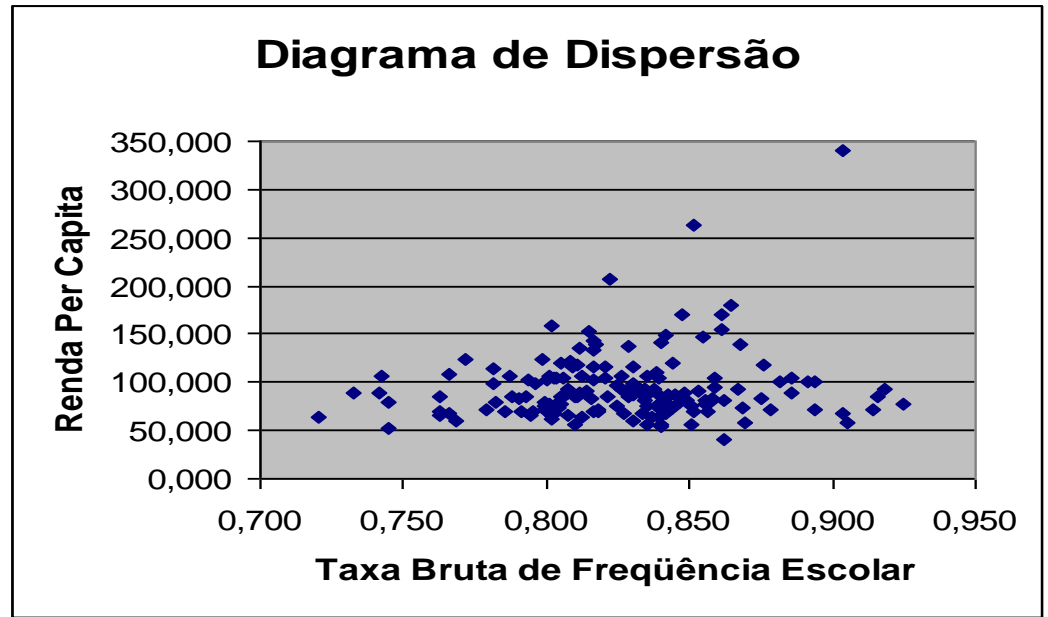

Fonte: Dados Próprios 
A correlação entre o IDH-M e a Renda per Capita dos 166 municípios, apresentou um resultado expressivo. A covariância (X, Y) foi de 35443,94, o desvio de X resultou em 7,110527, já o desvio de Y, em 5891,057. Esses resultados geram uma Correlação de 0,846149, um resultado considerável e que leva à afirmação de que o IDH-M têm influência considerável na Renda per Capita da população do Rio Grande do Norte.

O Gráfico 9 ilustra a Correlação verificada, positiva e linear, com um diagrama de dispersão.

Gráfico 9: Diagrama de Dispersão da Correlação entre IDH-M e Renda Per Capita.

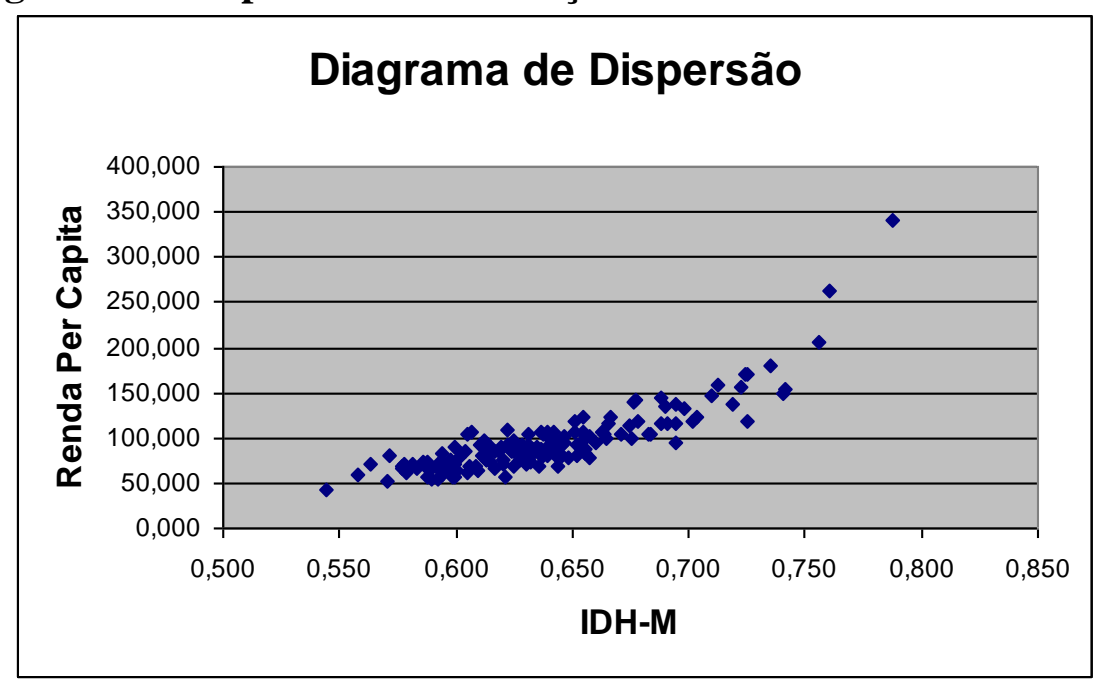

Fonte: Dados Próprios

O grau de Explicação dessa relação é de aproximadamente 71,6\%. Isto é, 71,6\% da variação da variável da Renda per Capita da população do Rio Grande do Norte pode ser explicada pela variação da variável IDH-M.

Ademais, com base nessa Explicação, pode-se ajustar uma função de Regressão para expressar essa relação. A equação, então, é a seguinte:

$Y_{p}=91,86887+3,160243 . X$.

Ou seja, caso tenhamos interesse, é possível estimar valores para a variável Y- IDH-M a partir de valores da variável X-Renda per Capita. Deste modo, caso uma Cidade "Z" do rio Grande do Norte tivesse um valor de IDH-M igual a 0,700, o valor de sua Renda per Capita seria, muito provavelmente, em torno de 94,0810401 reais.

Seguindo, calculamos o erro padrão da estimativa, que mede o quanto há de erro em uma estimativa de regressão. Cada previsão estará sujeita a esse erro, de 35,58887 reais, para mais ou para menos.

Finalmente, para essa Cidade " $Z$ ”, aplicando o Erro-padrão à estimativa da Renda per Capita de 94,0810401 reais, para mais e para menos, poder-se-ia afirmar que a Renda per Capita variaria de 58,49217 reais a reais 129,6699 . 


\section{CONSIDERAÇÕES FINAIS E RECOMENDAÇÕES}

Uma análise dos resultados permite-nos concluir que apenas uma correlação foi considerada forte. Isso ocorreu entre os dados do IDH e da Renda per Capita. Diante disso, constatamos que o IDH é responsável por explicar aproximadamente $71,6 \%$ da variação da renda per capita.

Dos 18 municípios que ultrapassaram a média da Renda per Capita estadual, um total de 16 também ultrapassaram a média do IDH-M. Foram eles, com seus respectivos valores de Renda per Capita e de IDH-M:

Acari (133,382 e 0,698), Alto do Rodrigues (143,884 e 0,688), Areia Branca (146,308 e 0,710),

Caicó (206,643 e 0,756), Carnaúba dos Dantas (152,896 e 0,742), Cruzeta (158,764 e 0,713),

Currais Novos (170,802 e 0,713), Extremoz (136,497 e 0,695), Jardim do Seridó (155,207 e 0,722),

Macau (135,898 e 0,690), Mossoró (179,590 e 0,735), Natal (339,923 e 0,787),

Parnamirim (263,008 e 0,760), Pau dos Ferros (171,090 e 0,725), São José do Seridó (149,647 e 0,740) e Timabúba dos Batistas (138,353 e 0,719).

Contrapondo-se a esse quadro muito bom de IDH-M e Renda per Capita, temos municípios que comprovam a Correlação entre aqueles em seus piores resultados. Assim, das 7 Cidades piores colocadas, ou seja, abaixo da média, em Renda per Capita no Estado, um total de 5 também encontram-se nas colocações inferiores de IDH-M, são elas, acompanhadas de seus respectivos índices de Renda per Capita e de IDH-M:

Água Nova (56,583 e 0,588), Coronel João Pessoa (55,230 e 0,592), Japi (51,760 e 0,570), João Dias (53,533 e 0,590) e Venha-Ver (41,551 e 0,544).

A pior situação, como pode-se observar, é do Município de Venha-Ver, Cidade no interior do Rio Grande do Norte, com uma população de 2.493 habitantes.

O município de Natal, como já era esperado, obtém os dois maiores valores; enquanto o município de Parnamirim, se forem levadas em consideração somente essas duas variáveis, coloca-se como a segunda Cidade do estado. Mossoró, coloca-se apenas como quarta cidade, atrás ainda de Caicó. Esta situação pode ser melhor visualizada na Figura 1. 


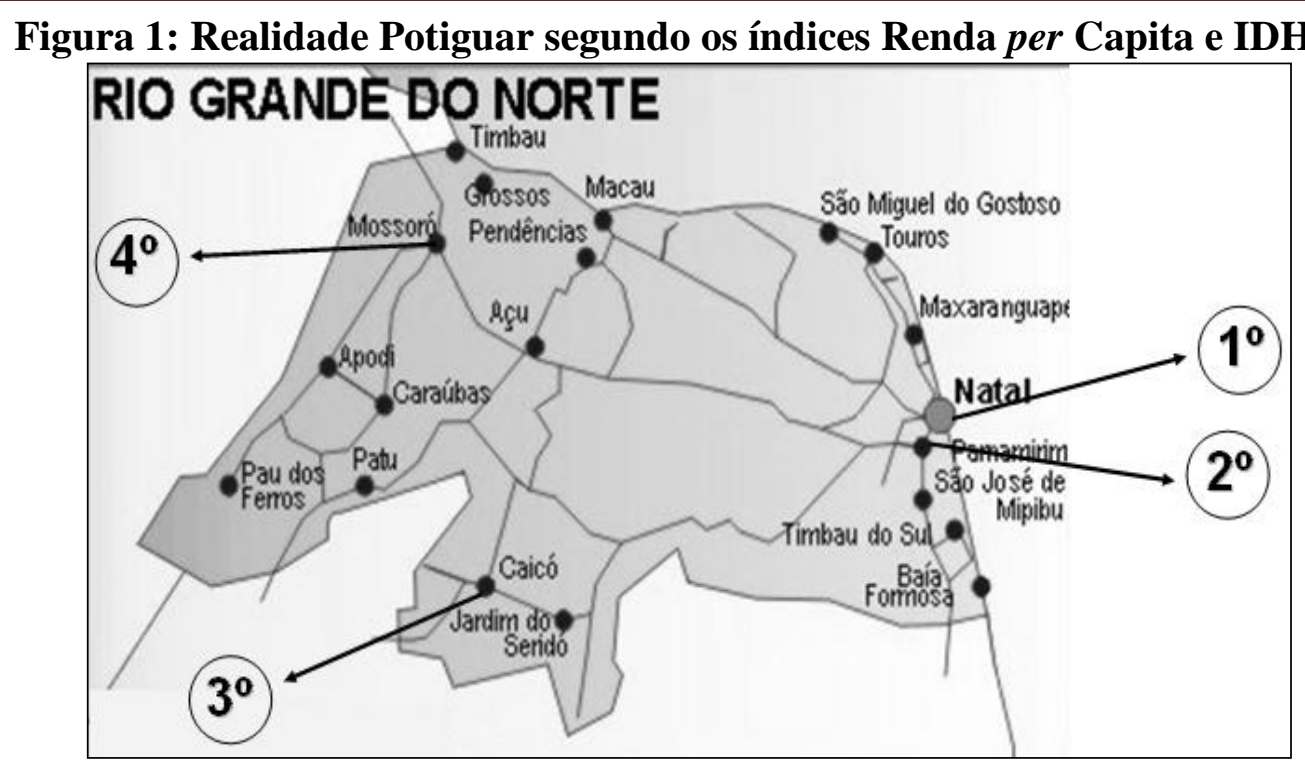

Fonte: Dados Próprios

Segundo o site Internet Produto Interno Bruto (www.ipib.com.br), o Estado do Rio Grande do Norte, como um todo, localiza-se na $19^{\circ}$ colocação na classificação dos Estados Brasileiros no quesito Renda per Capita, com 4.039,00 reais. Quanto ao IDH, ele está em $10^{\circ}$ lugar. Esses dados são de 2002. Portanto, o que verificamos foram valores bastante reduzidos das três variáveis para todos os municípios do estado do $\mathrm{RN}$.

Diante desses resultados, que nos proporcionaram uma visão mais crítica das situações social, econômica e educacional do nosso Estado do Rio Grande do Norte, podemos, agora, tecer sugestões e recomendações, tanto para futuros trabalhos, como para interessados no tema.

Propomos, assim, como forma de complementação do nosso estudo, trabalhos que:

- possam abordar os principais fatores que interferem na má ou boa qualidade das variáveis analisadas; analisem a interferência das variáveis estudadas na contribuição do Estado para o PIB Nacional;

- façam um estudo mais apurado da contribuição das classes econômicas do estado para a formação da nossa Renda per Capita;

- utilizem-se de mais variáveis no intuito de captar as principais Correlações existentes entre elas.

Pudemos perceber também investimentos no campo social para melhorar o IDH-M do nosso Estado, no intuito de melhorar também os valores de Renda per Capita da população, devem ser, inquestionavelmente, defendidos por toda nossa população e todas as esferas Governamentais.

\section{REFERÊNCIAS}

1. ALBUQUERQUE, Moisés. Mossoró está na lista das $\mathbf{1 0 0}$ melhores para fazer carreira. Correio da Tarde. 386 ed., Ano II. Natal e Mossoró: 24 jul. 2007. Disponível em: 
<<http://www.correiodatarde.com.br/colunistas/moises_albuquer_-19631>>. Acesso em: 06 jan. 2008.

2. BATELLA, Wagner B.; DINIZ, Alexandre M. A. Desenvolvimento humano e hierarquia urbana: uma análise do IDH-M entre as cidades mineiras. Revista de Biologia e Ciências da Terra. v. 6. n. 2. 2006. p. 367-374.

3. BREMAEKER, François E. J. de. O Índice de Desenvolvimento Humano dos Municípios das Capitais em 2000. Coleção Estudos da Cidade. Prefeitura da Cidade do Rio de Janeiro, SMU, IPP, DIG: jan, 2003.

4. CRESPO, Antônio Arnot. Estatística Fácil. 18. ed. São Paulo: Saraiva, 2002.

5. DUARTE, Angelo J. M.; FERREIRA, Pedro C.; SALVATO, Márcio A. Disparidades Regionais ou Educacionais? Um Exercício Confractual. In: TEIXEIRA, E.C.; BARBOSA, M.J. (Org.). Investimento e Crescimento Econômico no Brasil. 1 ed. Viçosa: Editora da UFV, 2006, v. 1, p. 333-369.

6. FONSECA, Jairo Simon da; MARTINS, Gilberto de Andrade. Curso de Estatística. 6 ed. São Paulo: Atlas, 1996.

7. ÍNDICE de Desenvolvimento Humano Municipal (IDH-M) 2000. Disponível em: <<http://www.frigoletto.com.br/GeoEcon/idhrn.htm>>. Acesso em: 06 jul. 2007.

8. INTERNET Produto Interno Bruto. Disponível em: <<www.ipib.com.br〉>. Acesso em: 27 jul. 2007.

9. GIL, Antonio Carlos. Como Elaborar Projetos de Pesquisa. 4. ed. São Paulo: Atlas, 2002.,

10. LEONARDI, Alexi. Desenvolvimento Social na América Latina e sua relação com o crescimento econômico na década de 1990. Universidade Federal de Santa Maria, 2005. V. 3, 89p. Disponível em: << http://www.capes.gov.br/>>. Acesso em: 02 out. 2007.

11. LUCCI, Elian Alabi; BRANCO, Anselmo Lazaro; MENDONÇA, Cláudio. Geografia Geral e do Brasil. 2 ed. São Paulo: Saraiva, 2004.

12. MAIA, Lerson Fernando dos Santos; OLIVEIRA, Marcus Vinícius de Faria. Trabalhos Acadêmicos: princípios, normas e técnicas. Natal: CEFET/RN, 2006.

13. MARCOLINO, César R. F. Renda per Capita e Índice de Desenvolvimento Humano no Brasil. Universidade Federal de Viçosa, 2002. 94p. Disponível em: <<http://www.capes.gov.br/>>. Acesso em: 02 out. 2007.

14. MOORE, David S.; MCCABE, George P. Introdução à Prática da Estatística. $3^{\mathrm{a}}$ ed. Rio de Janeiro: Editora LTC, 2002.

15. MOSSORÓ tem apenas o sexto melhor IDH do RN. Jornal O Mossoroense. 30 jul. 2003. Disponível em: <<http://www2.uol.com.br/omossoroense/051003/cotidiano3.htm〉>. Acesso em: 28 jul. 2007.

16. PROGRAMA DAS NAÇÕES UNIDAS PARA O DESENVOLVIMENTO - PNUD (Org.). Atlas do Desenvolvimento Humano no Brasil - 2003: Entenda o cálculo do IDH Municipal (IDH-M). Disponível em: <http://64.233.169.104/search?q=cache:Cv1nBrgV60wJ:www.pnud.org.br/atlas/PR/Calcul o_IDH.doc+\%22O+segundo+indicador+\%C3\%A9+resultado+de+uma+conta+simples:+o +somat $\% \mathrm{C} 3 \%$ B3rio $\% 22 \& \mathrm{hl}=\mathrm{pt}-\mathrm{BR} \& \mathrm{ct}=\mathrm{clnk} \& \mathrm{~cd}=1 \& \mathrm{gl}=\mathrm{br} \& \mathrm{client}=$ firefox-a $>$. Acesso em: 26 jul. 2007. 
17. RENDA per capita cresce, mas consumo perde participação. Do Rio. Valor Econômico, 012 abr. 2007.2 Disponível em: $<<$ http://clipping.planejamento.gov.br/Noticias.asp?NOTCod= 186233〉>. Acesso em: 28 jul. 2007.

18. SOUZA, Luciana S.; SANTOS, Haroldo E. A. dos; SOUZA, Romina B. de L. de. O Desenvolvimento Humano do Município de Boa Vista, RR. Análise. v. 19. n. 2. Porto Alegre/RS: jul/dez, 2008. p. 78-92.

19. SPIEGEL, Murray R. Estatística. Tradução e revisão técnica - Pedro Consentino. 3 ed. São Paulo: Makorn Books, 1993.

20. WIKIPÉDIA, A enciclopédia livre. Renda per capita. Disponível em: <<http://pt.wikipedia.org/wiki/Renda_per_capita〉>. Acesso em: 26 jul. 2007. 